\title{
Discovery of very high energy $\gamma$-ray emission from the BL Lacertae object H 2356-309 with the HESS Cherenkov telescopes
}

\author{
F. Aharonian ${ }^{1}$, A. G. Akhperjanian ${ }^{2}$, A. R. Bazer-Bachi ${ }^{3}$, M. Beilicke ${ }^{4}$, W. Benbow ${ }^{1}$, D. Berge ${ }^{1}$, K. Bernlöhr ${ }^{1,5}$,
}

C. Boisson ${ }^{6}$, O. Bolz ${ }^{1}$, V. Borrel ${ }^{3}$, I. Braun ${ }^{1}$, F. Breitling ${ }^{5}$, A. M. Brown ${ }^{7}$, R. Bühler ${ }^{1}$, I. Büsching ${ }^{8}$, S. Carrigan ${ }^{1}$, P. M. Chadwick ${ }^{7}$, L.-M. Chounet ${ }^{9}$, R. Cornils ${ }^{4}$, L. Costamante ${ }^{1,21}$, B. Degrange ${ }^{9}$, H. J. Dickinson ${ }^{7}$, A. Djannati-Atai ${ }^{10}$, L. O'C. Drury ${ }^{11}$, G. Dubus ${ }^{9}$, K. Egberts ${ }^{1}$, D. Emmanoulopoulos ${ }^{12}$, P. Espigat ${ }^{10}$, F. Feinstein ${ }^{13}$, E. Ferrero ${ }^{12}$, G. Fontaine ${ }^{9}$, Seb. Funk ${ }^{5}$, S. Funk ${ }^{1}$, Y. A. Gallant ${ }^{13}$, B. Giebels ${ }^{9}$, J. F. Glicenstein ${ }^{14}$, P. Goret ${ }^{14}$, C. Hadjichristidis ${ }^{7}$, D. Hauser ${ }^{1}$, M. Hauser ${ }^{12}$, G. Heinzelmann ${ }^{4}$, G. Henri ${ }^{15}$, G. Hermann ${ }^{1}$, J. A. Hinton ${ }^{1,12}$, W. Hofmann ${ }^{1}$, M. Holleran ${ }^{8}$, D. Horns ${ }^{16}$, A. Jacholkowska ${ }^{13}$, O. C. de Jager ${ }^{8}$, B. Khélifi ${ }^{9,1}$, Nu. Komin ${ }^{5}$, A. Konopelko ${ }^{5}$, I. J. Latham ${ }^{7}$,

R. Le Gallou ${ }^{7}$, A. Lemière ${ }^{10}$, M. Lemoine-Goumard ${ }^{9}$, T. Lohse ${ }^{5}$, J. M. Martin ${ }^{6}$, O. Martineau-Huynh ${ }^{17}$, A. Marcowith $^{3}$, C. Masterson ${ }^{1,21}$, T. J. L. McComb ${ }^{7}$, M. de Naurois ${ }^{17}$, D. Nedbal ${ }^{18}$, S. J. Nolan ${ }^{7}$, A. Noutsos ${ }^{7}$, K. J. Orford ${ }^{7}$, J. L. Osborne ${ }^{7}$, M. Ouchrif ${ }^{17,21}$, M. Panter ${ }^{1}$, G. Pelletier ${ }^{15}$, S. Pita ${ }^{10}$, G. Pühlhofer ${ }^{12}$, M. Punch ${ }^{10}$, B. C. Raubenheimer ${ }^{8}$, M. Raue ${ }^{4}$, S. M. Rayner ${ }^{7}$, A. Reimer ${ }^{19}$, O. Reimer ${ }^{19}$, J. Ripken ${ }^{4}$, L. Rob ${ }^{18}$, L. Rolland ${ }^{14}$, G. Rowell ${ }^{1}$, V. Sahakian ${ }^{2}$, L. Saugé ${ }^{15}$, S. Schlenker ${ }^{5}$, R. Schlickeiser ${ }^{19}$, U. Schwanke ${ }^{5}$, H. Sol ${ }^{6}$, D. Spangler ${ }^{7}$, F. Spanier ${ }^{19}$, R. Steenkamp ${ }^{20}$, C. Stegmann ${ }^{5}$, G. Superina ${ }^{9}$, J.-P. Tavernet ${ }^{17}$, R. Terrier ${ }^{10}$, C. G. Théoret ${ }^{10}$, M. Tluczykont ${ }^{9,21,22}$, C. van Eldik ${ }^{1}$, G. Vasileiadis ${ }^{13}$, C. Venter ${ }^{8}$, P. Vincent ${ }^{17}$, H. J. Völk ${ }^{1}$, S. J. Wagner ${ }^{12}$, and M. Ward ${ }^{7}$

(Affiliations can be found after the references)

Received 20 December 2005 / Accepted 7 May 2006

\section{ABSTRACT}

\begin{abstract}
The extreme synchrotron BL Lac object H 2356-309, located at a redshift of $z=0.165$, was observed from June to December 2004 with a total exposure of $\approx 40 \mathrm{~h}$ live-time with the HESS (High Energy Stereoscopic System) array of atmospheric-Cherenkov telescopes (ACTs). Analysis of this data set yields, for the first time, a strong excess of $453 \gamma$-rays (10 standard deviations above background) from H 2356-309, corresponding to an observed integral flux above $200 \mathrm{GeV}$ of $I(>200 \mathrm{GeV})=(4.1 \pm 0.5) \times 10^{-12} \mathrm{~cm}^{-2} \mathrm{~s}^{-1}$ (statistical error only). The differential energy spectrum of the source between $200 \mathrm{GeV}$ and $1.3 \mathrm{TeV}$ is well-described by a power law with a normalisation (at $1 \mathrm{TeV})$ of $N_{0}=\left(3.00 \pm 0.80_{\text {stat }} \pm\right.$ $\left.0.31_{\text {sys }}\right) \times 10^{-13} \mathrm{~cm}^{-2} \mathrm{~s}^{-1} \mathrm{TeV}^{-1}$ and a photon index of $\Gamma=3.09 \pm 0.24_{\text {stat }} \pm 0.10_{\text {sys. }}$. H 2356-309 is one of the most distant BL Lac objects detected at very-high-energy $\gamma$-rays so far. Results from simultaneous observations from ROTSE-III (optical), RXTE (X-rays) and NRT (radio) are also included and used together with the HESS data to constrain a single-zone homogeneous synchrotron self-Compton (SSC) model. This model provides an adequate fit to the HESS data when using a reasonable set of model parameters.
\end{abstract}

Key words. gamma rays: observations - galaxies: active - galaxies: BL Lacertae objects: individual: H 2356-309

\section{Introduction}

The Spectral Energy Distribution (SED) of Active Galactic Nuclei (AGN) spans the complete electromagnetic spectrum from radio waves to very-high-energy (VHE; $E>100 \mathrm{GeV}$ ) $\gamma$-rays. In the widely-accepted unified model of AGN (e.g. Rees 1984; Urry \& Padovani 1995), the "central engine" of these objects consists of a super-massive black hole (up to $10^{9} M_{\odot}$ ) surrounded by a thin accretion disk and a dust torus. In some radio-loud $A G N$, i.e. objects with a radio to $B$-band flux ratio $F_{5 \mathrm{GHz}} / F_{B}>10$, two relativistic plasma outflows (jets) presumably perpendicular to the plane of the accretion disk have been observed.

AGN are known to be VHE $\gamma$-ray emitters since the detection of Mrk 421 above $300 \mathrm{GeV}$ by the Whipple group (Punch et al. 1992), who pioneered the imaging atmospheric-Cherenkov technique. At very high energies, a number of AGN $(\approx 10)$ were subsequently detected by different groups using a similar technique. Almost all these objects are BL Lacertae (BL Lac) objects, belonging to the class of Blazars (BL Lac objects and Flat
Spectrum Radio Quasars), i.e. AGN having their jet pointing at a small angle to the line of sight. The only confirmed VHE detection of an extragalactic object not belonging to the BL Lac class is the giant radio galaxy M 87 (Aharonian et al. 2003, 2005d).

Two broad peaks are present in the observed SED of AGN. The first peak is located in the radio, optical, and X-ray bands, the second peak is found at higher energies and can extend to the VHE band. The observed broad-band emission from AGN is commonly explained by two different model types. In leptonic models, the lower-energy peak is explained by synchrotron emission of relativistic electrons and the high-energy peak is assumed to result from inverse Compton (IC) scattering of electrons off a seed-photon population, see e.g. Sikora \& Madejski (2001) and references therein. In hadronic models, the emission is assumed to be produced via the interactions of relativistic protons with matter (Pohl \& Schlickeiser 2000), ambient photons (Mannheim 1993) or magnetic fields (Aharonian 2000), or via the interactions of relativistic protons with photons and magnetic fields (Mücke \& Protheroe 2001). 
The observed $\gamma$-ray emission from BL Lac objects shows high variability ranging from short bursts of sub-hour duration to long-time activity of the order of months. Detailed studies of variability of BL Lac type objects can contribute to the understanding of their intrinsic acceleration mechanisms (e.g. Krawczynski et al. 2001; Aharonian et al. 2002). Additionally, observations of distant objects in the VHE band provide an indirect measurement of the SED of the Extragalactic Background Light (EBL), see e.g. Stecker et al. (1992); Primack et al. (1999) and references therein. Due to the absorption of VHE $\gamma$-rays via $\mathrm{e}^{+} \mathrm{e}^{-}$pair production with the photons of the EBL, the shape of the observed VHE spectra is distorted as compared to the intrinsically emitted spectra. Using a given spectral shape of the EBL, the observed AGN spectrum can be corrected for this absorption. The resulting intrinsic (i.e., corrected) spectrum can then be compared to basic model assumptions on the spectral shape of the $\gamma$-ray emission, thereby constraining the applied shape of the EBL. In this context, it is especially important to detect AGN at higher redshifts but also to study the spectra of objects over a wide range of redshifts, in order to disentangle the effect of the EBL from the intrinsic spectral shape of the objects. To date, the redshifts of VHE emitting BL Lac objects with measured spectra range from $z=0.033$ to $z=0.129$.

The high frequency peaked BL Lac object (HBL) H 2356-309, identified in the optical by Schwartz et al. (1989), is hosted by an elliptical galaxy located at a redshift of $z=$ 0.165 (Falomo 1991). The object was first detected in X-rays by the satellite experiment UHURU (Forman et al. 1978) and subsequently by the Large Area Sky Survey experiment onboard the HEAO-I satellite (Wood et al. 1984). The spectrum of H 2356-309 as observed by BeppoSAX (Costamante et al. 2001) is not compatible with a single power law model, indicating that the peak of the synchrotron emission lies within the energy range of BeppoSAX. A broken power law fit yields a synchrotron peak around $1.8 \mathrm{keV}$, with a detection of the source up to $50 \mathrm{keV}$. These observations qualified the object as an extreme synchrotron blazar.

A selection of $\mathrm{TeV}$ candidate BL Lac objects was proposed by Costamante $\&$ Ghisellini (2002). The objects were selected from several BL Lac samples and using information in the radio, optical and X-ray bands. VHE predictions for the selected objects were given by the authors based on a parametrisation proposed by Fossati et al. (1998), suitable for predictions of high state flux of an average source population. The authors also gave VHE flux predictions based on a simple one-zone homogeneous SSC model (Ghisellini et al. 2002), appropriate for a quiescent state of the specific VHE source candidate. H2356-309 is included in this list and the predicted integral flux values above $300 \mathrm{GeV}$ for $\mathrm{H} 2356-309$ are $8.4 \times 10^{-12} \mathrm{~cm}^{-2} \mathrm{~s}^{-1}$ for the parametrisation and $1.9 \times 10^{-12} \mathrm{~cm}^{-2} \mathrm{~s}^{-1}$ for the SSC model. It should be noted that no absorption due to the EBL was taken into account in these calculations.

In this paper the discovery of VHE $\gamma$-rays from H 2356-309 with the HESS Cherenkov telescopes in 2004 is reported. With a redshift of $z=0.165, \mathrm{H} 2356-309$ is one of the most distant AGN detected at VHE energies so far. H2356-309 was observed by HESS from June to December 2004 (see Sects. 2 and 3). Simultaneous observations were carried out with RXTE (Rossi X-ray Timing Explorer) in X-rays on 11th of November 2004 (see Sect. 4.1), with the Nançay decimetric radio telescope (NRT) between June and October 2004 (see Sect. 4.2) and with ROTSE-III (see Sect. 4.3) in the optical, covering the whole 2004 HESS observation campaign.

\section{HESS observations}

The system of four HESS ACTs, located on the Khomas Highlands in Namibia $\left(23^{\circ} 16^{\prime} 18^{\prime \prime} \mathrm{S} 16^{\circ} 30^{\prime} 00^{\prime \prime} \mathrm{E}\right)$, is fully operational since December 2003. For a review see, e.g., Hinton (2004). HESS data are taken in runs with a typical duration of 28 min. The data on H2356-309 were taken with the telescopes pointing with an offset of $0.5^{\circ}$ relative to the object position (wobble mode, offset in either right ascension or declination). The sign of the offset is alternated for successive runs to reduce systematic effects. H 2356-309 was observed with the complete stereoscopic system from June to December 2004 for a total raw observation time of more than $80 \mathrm{~h}$. In order to reduce systematic effects that arise due to varying observation conditions, quality selection criteria are applied before data analysis on a run-by-run basis. The criteria are based on the mean trigger rate (corrected for zenith-angle dependency), trigger rate stability, weather conditions and hardware status. During the 2004 observations of H 2356-309 atmospheric conditions were not optimal (due to brushfires), resulting in a dead-time corrected high-quality data set of $\approx 40 \mathrm{~h}$ live-time at an average zenith angle of $20^{\circ}$.

These data are calibrated as described in Aharonian et al. (2004). Thereafter, before shower reconstruction, a standard image cleaning (Lemoine-Goumard et al. 2005) is applied to the shower images to remove night-sky background noise. Moreover, in order to avoid systematic effects from shower images truncated by the camera edge, only images having a distance between their centre of gravity and the centre of the camera of less than $2^{\circ}$ are used in the reconstruction. Furthermore, a minimum image amplitude (i.e., the sum of the intensities of all pixels being part of the image) is required for use in the analysis to assure a good quality reconstruction. Previous HESS publications are mostly based on the standard analysis (Aharonian et al. 2005a). Here we present results from the 3D Model analysis which is presented in detail in Lemoine-Goumard et al. (2005) and was also used in Aharonian et al. (2005b). This method uses independent calibration and simulation chains and is briefly described in the following paragraphs.

\subsection{HESS 3D model analysis}

The principle of the 3D Model reconstruction method (Lemoine-Goumard \& Degrange 2004; Lemoine-Goumard et al. 2005; Aharonian et al. 2005b) is based on a 3-dimensional (3D) shower model using the stereoscopic information from the telescopes. The shower is modelled as a 3D Gaussian photosphere with anisotropic angular distribution. For each camera pixel the expected light is calculated with a path integral along the line of sight. The observed images are then compared to the model images using a log-likelihood fit with eight parameters, described in detail in Lemoine-Goumard et al. (2005). For each detected shower at least two images are required for the reconstruction of the angle $\theta$ (the angle between the object position and the reconstructed shower direction), shower core impact position (measured as a radius with respect to the center of the telescope array), energy and the transverse standard deviation $\sigma_{\mathrm{T}}$ of the shower. The dimensionless reduced 3D width $\omega$ (used for $\gamma$-hadron separation) is defined as $\omega=\sigma_{\mathrm{T}} \rho / D_{\mathrm{S}}$, with the density of air $\rho$ and the column density $D_{\mathrm{S}}$ at shower maximum. The energy spectrum of the $\gamma$-ray excess is obtained from a comparison of the reconstructed energy distributions to the expected distributions for a given spectral shape. For the determination of the expected number, $\gamma$-ray acceptances calculated from simulations 
Table 1. Summary of applied cuts. The cut in image amplitude is given in photoelectrons (ph.e.).

\begin{tabular}{ll}
\hline \hline Cut & Value \\
\hline Distance & 2 deg \\
Image amplitude & $>60$ ph.e. \\
\# of telescopes & $\geq 3$ \\
Core impact position & $\leq 300 \mathrm{~m}$ \\
Mean reduced scaled width & - \\
Mean reduced scaled length & - \\
Reduced 3D width $\omega$ & $<0.002$ \\
$\theta^{2}$ & $<0.01 \mathrm{deg}^{2}$ \\
\hline
\end{tabular}

are taken into account. This forward folding method was first developed within the CAT collaboration (Piron 2000). The quality of the reconstruction improves when only events with a number of triggered telescopes $N_{\text {tel }} \geq 3$ are accepted. In the analysis used in this paper, results from the 3D Model are given using this cut. A spectral analysis using an $N_{\text {tel }} \geq 2$ cut yields consistent results.

The on-source data are taken from a circular region of radius $\theta$ around the object position (on-source region). The background is estimated from 11 control regions (off-source regions) of the same size and located at the same radial distance to the camera centre as the on-source region and normalised accordingly. The significance in standard deviations above background (subsequently $\sigma$ ) of any excess is calculated following the likelihood method of Li \& Ma (1983). All cuts applied are summarised in Table 1. Cut values were optimised using simulated $\gamma$-ray samples and independent background data samples.

\section{HESS results}

\subsection{Signal}

In Fig. 1, the distribution of the squared angular distances $\theta^{2}$ from H 2356-309, reconstructed with the 3D-Model $\left(N_{\text {tel }} \geq 3\right.$ ), is shown. In the data taken from the on-source region a clear accumulation of events is seen at low $\theta^{2}$-values, i.e. close to the position of H2356-309. The off-region shows a flat distribution as expected for a pure background measurement. With a number of on-source events of $N_{\text {on }}=1706$ off-source events $N_{\text {off }}=13784$ and a normalisation factor $\alpha=0.0909$ (the ratio between the solid angles for on- and off-source measurements), the data yield an excess of $N_{\text {on }}-\alpha N_{\text {off }}=453 \gamma$-rays at a significancelevel of $11.6 \sigma$. A fit of a 2-dimensional Gaussian to an uncorrelated excess sky-map yields a point-like emission and a location $\left(23^{\mathrm{h}} 59^{\mathrm{m}} 09.42^{\mathrm{s}} \pm 2.89^{\mathrm{s}},-30^{\circ} 37^{\prime} 22.7^{\prime \prime} \pm 34.5^{\prime \prime}\right)$ consistent with the position of $\mathrm{H} 2356-309\left(23^{\mathrm{h}} 59^{\mathrm{m}} 07.8^{\mathrm{s}},-30^{\circ} 37^{\prime} 38^{\prime \prime}\right)$, as obtained by Falomo (1991) using observations in the optical and near-infrared. These results are summarised in Table 2. Additionally, the results from the standard analysis are given. The results for the 3D Model analysis used in this paper are also given using $N_{\text {tel }} \geq 2$ for easier comparison with the standard analysis.

\subsection{Energy spectrum and variability}

The differential energy spectrum obtained from the 3D-Model analysis $\left(N_{\text {tel }} \geq 3\right)$ is shown in Fig. 2. The spectral parameters were obtained from a maximum likelihood fit of a power law hypothesis $\mathrm{d} N / \mathrm{d} E=N_{0}(E / \mathrm{TeV})^{-\Gamma}$ to the data, resulting in a flux-normalisation of $N_{0}=(3.00 \pm$ $\left.0.80_{\text {stat }} \pm 0.31_{\text {sys }}\right) \times 10^{-13} \mathrm{~cm}^{-2} \mathrm{~s}^{-1} \mathrm{TeV}^{-1}$, and a spectral index

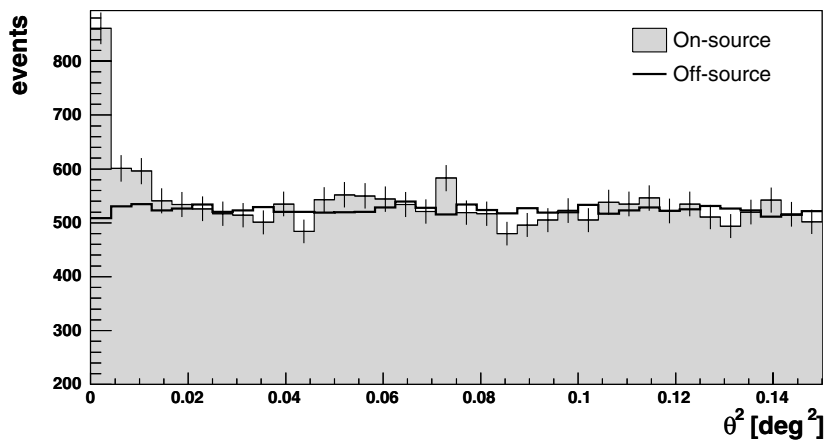

Fig. 1. Distribution of squared angular distances $\theta^{2}$ for H2356-309, using the 3D-Model reconstruction and $N_{\text {tel }} \geq 3$.

Table 2. Summary of analysis results from the 3D Model analysis (using two different cuts in telescope multiplicity). For comparison, the standard analysis results are also given.

\begin{tabular}{llll}
\hline \hline & \multicolumn{2}{c}{ 3D Model } & standard analysis \\
\hline & $N_{\text {tel }} \geq 3$ & $N_{\text {tel }} \geq 2$ & $N_{\text {tel }} \geq 2$ \\
$N_{\text {on }}$ (events) & 1706 & 4389 & 3776 \\
$N_{\text {off }}$ (events) & 13784 & 40420 & 35280 \\
normalisation $\alpha$ & 0.0909 & 0.0909 & 0.0903 \\
Excess (events) & 453 & 715 & 591 \\
Significance & $11.6 \sigma$ & $10.9 \sigma$ & $9.7 \sigma$ \\
\hline
\end{tabular}

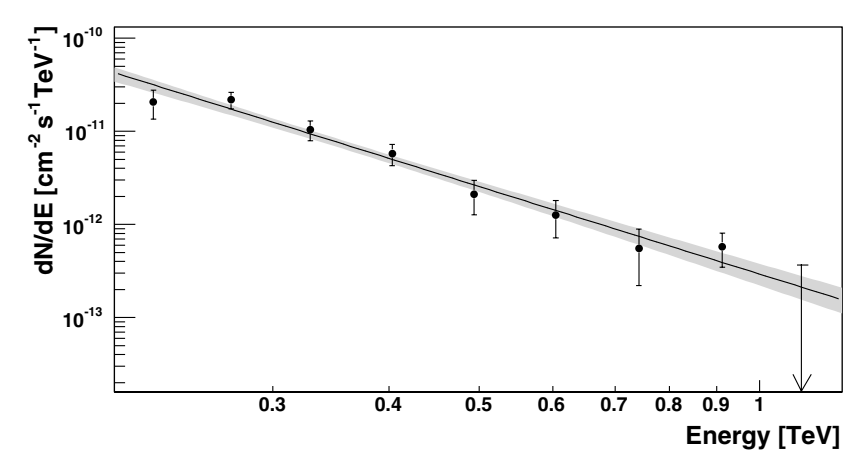

Fig. 2. Differential energy spectrum of H2356-309, as obtained from the 3D Model analysis. The shaded area gives the confidence region (1 standard deviation) for the spectral shape under the assumption of a power law. The upper limit (arrow) is given with a 99\% confidence level.

of $\Gamma=3.09 \pm 0.24_{\text {stat }} \pm 0.10_{\text {sys }}$. The $\chi^{2}$ value of the spectral fit is 6.6 for 7 degrees of freedom, corresponding to a $\chi^{2}$-probability of $P\left(\chi^{2}\right)=0.47$. In order to eliminate any systematic effects that might arise from poor energy estimation at lower energies (due to an over-estimation, on average, of low energies), the beginning of the fit range is set to the value of the post-cuts spectral energy threshold, i.e. $200 \mathrm{GeV}$ for the 3D Model analysis of this data set. Systematic errors ( 0.1 for the index and $20 \%$ for the flux) are dominated by atmospheric effects, i.e. a limited knowledge of the atmospheric profile needed as input for the simulations. A detailed description of systematic errors can be found in e.g., Aharonian et al. (2006b). The parameters of the spectral fit are summarised in Table 3. Additionally, the results from the standard analysis are given for comparison. The data-points used in Fig. 2 are listed in Table 4.

The average integral flux above $200 \mathrm{GeV}$ in the year 2004 (fitting with a fixed spectral index of 3.09) is $\overline{I(>200 \mathrm{GeV})}=(4.1 \pm 0.5) \times 10^{-12} \mathrm{~cm}^{-2} \mathrm{~s}^{-1} \quad$ (statistical error only). Light-curves of $I(>200 \mathrm{GeV})$ versus the modified Julian 
Table 3. Summary of the parameters of power law fits $(\mathrm{d} N / \mathrm{d} E=$ $\left.N_{0}(E / \mathrm{TeV})^{-\Gamma}\right)$. For comparison with the 3D Model analysis (1) used here, the results from the standard analysis (2) are also given. Both analyses yield consistent results. In addition to the parameter values, the $\chi^{2}$ probabilities of the fits are given.

\begin{tabular}{cll}
\hline \hline$\Gamma$ & $N_{0}$ & $P\left(\chi^{2}\right)$ \\
& {$\left[10^{-13} \mathrm{~cm}^{-2} \mathrm{~s}^{-1} \mathrm{TeV}^{-1}\right]$} & \\
\hline (1) $3.09 \pm 0.24_{\text {stat }} \pm 0.10_{\text {sys }}$ & $3.00 \pm 0.80_{\text {stat }} \pm 0.31_{\text {sys }}$ & 0.47 \\
(2) $3.06 \pm 0.21_{\text {stat }} \pm 0.10_{\text {sys }} 3.07 \pm 0.75_{\text {stat }} \pm 0.31_{\text {sys }}$ & 0.60 \\
\hline
\end{tabular}

Table 4. Differential flux for different energy bins. The upper limit is given for a confidence level of $99 \%$.

\begin{tabular}{lcc}
\hline \hline $\begin{array}{l}E \\
{[\mathrm{TeV}]}\end{array}$ & \multicolumn{2}{c}{$\Phi$} \\
{$\left[\mathrm{cm}^{-2} \mathrm{~s}^{-1} \mathrm{TeV}^{-1}\right]$}
\end{tabular}

date (MJD) of the observation are shown in Fig. 3 for two different time-scales. The monthly flux variation is shown in the upper panel and the average monthly flux from June to December of 2004 is shown in the lower panel. A fit of a constant yields no evidence for nightly variability $\left(P\left(\chi^{2}\right)=0.18\right)$.

The ASM (All Sky Monitor) shows no significant X-ray excess nor variability in the same monthly intervals.

\section{Multi-wavelength analysis and results}

\subsection{RXTE analysis}

The RXTE/PCA (Jahoda \& PCA Team 1996) observed H 2356-309 twice for a total of 5.4 ks on 11 November 2004 after a Target of opportunity was triggered on this target. Due to poor weather conditions, HESS-observations were not possible on the night of November 11, or on the 2 prior nights. However, the RXTE observations can be considered simultaneous to the HESS observation campaign of November. The STANDARD2 data were extracted using the ftools in the HEASOFT 6.0 analysis software package provided by NASA/GSFC and filtered using the RXTE Guest Observer Facility recommended criteria. Only the signals from the top layer (X1L and X1R) are used from the PCA. The average spectrum shown in the SED (Fig. 4) is derived by using PCU0, PCU2 and PCU3 data. The faintbackground model is used and only the 3-20 PHA channel range is kept in XSPEC v. 11.3.2, or approximately $2-10 \mathrm{keV}$. The column density is fixed to the Galactic value of $N_{\mathrm{H}}=1.33 \times$ $10^{20} \mathrm{~cm}^{-2}$ obtained from the PIMMS $\mathrm{nH}$ program ${ }^{1}$ and is used in an absorbed power law fit. This yields an X-ray photon in$\operatorname{dex} \Gamma_{X}=2.43 \pm 0.11$ and a flux of $9.7_{-1.3}^{+0.3} \times 10^{-12} \mathrm{erg} \mathrm{cm}^{-2} \mathrm{~s}^{-1}$ in the $2-10 \mathrm{keV}$ band. The $\chi^{2}$ of the fit is 11 for 14 degrees of freedom, or a $\chi^{2}$ probability $P\left(\chi^{2}\right)=0.68$. This flux level is approximately a factor of 3 lower than what was reported from BeppoSAX observations by Costamante et al. (2001) and with a softer photon index than what was observed above $2 \mathrm{keV}$.

\footnotetext{
${ }^{1}$ See http://legacy.gsfc. nasa.gov/Tools/w3pimms.html
}
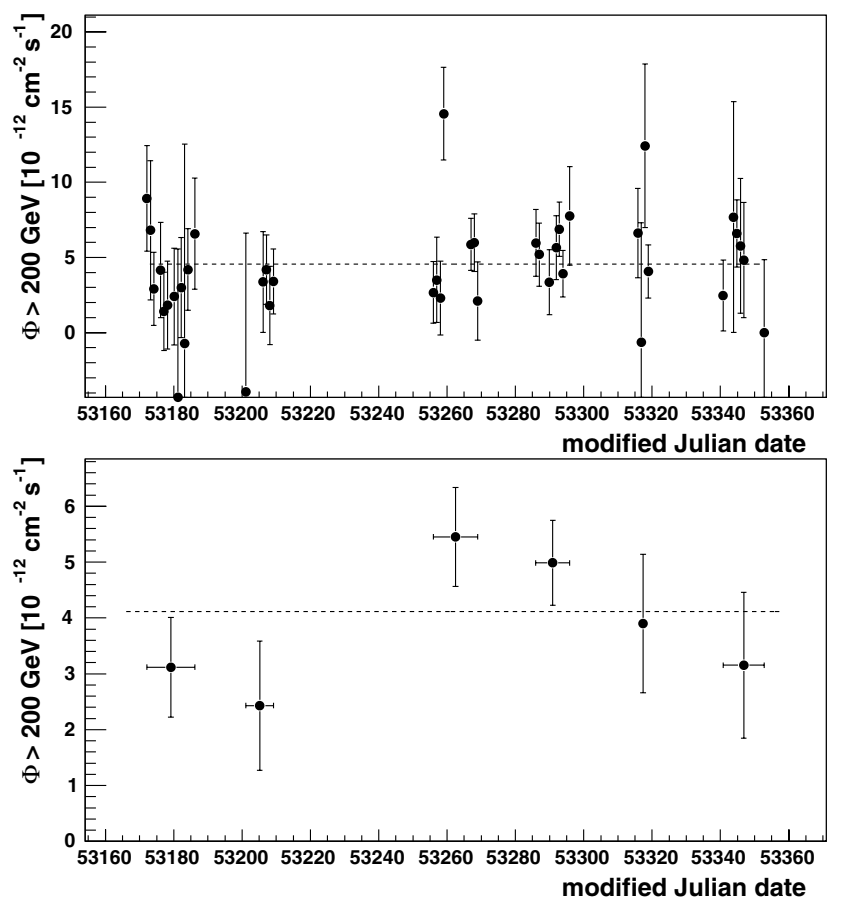

Fig. 3. Flux above $200 \mathrm{GeV}$ from H 2356-309 as measured by HESS (3D Model Analysis) in 2004. Upper panel: each point corresponds to the flux measurement in one night. Lower panel: averaged flux in six different time windows (June to December 2004). Both light-curves are consistent with a constant emission over time. The RXTE observations (see Sect. 4.1) were carried out on MJD 53320. During that night, no HESS observations could take place due to poor weather conditions.

\subsection{NRT analysis}

The Nançay radio-telescope is a single-dish antenna with a collecting area of $200 \times 34.56 \mathrm{~m}^{2}$ equivalent to that of a 94 m-diameter parabolic dish (van Driel et al. 1996). The halfpower beam width at $11 \mathrm{~cm}$ is $1.9 \operatorname{arcmin}(\mathrm{EW}) \times 11.5 \mathrm{arcmin}$ (NS) (at zero declination), and the system temperature is about $45 \mathrm{~K}$ in both horizontal and vertical polarisations. The point source efficiency is $0.8 \mathrm{~K} \mathrm{Jy}^{-1}$, and the chosen filter bandwidth was $12.5 \mathrm{MHz}$ for each polarisation, split into two subbands of $6.25 \mathrm{MHz}$ each. Data were processed using the Nançay local software NAPS and SIR.

A monitoring program with this telescope on extragalactic sources visible by both the NRT and HESS is in place since 2001. For the campaign described here it consisted of a measurement at $11 \mathrm{~cm}$ every two or three days. Between 4 and 14 individual 1-min drift scans were performed for each observation, and the flux calibration was done using a calibrated noise diode emission for each drift scan. The average flux for the measurements carried out between 11 June and 10 October 2004 was $40 \pm 8 \mathrm{mJy}$. This observed flux is most likely dominated by emission produced in jet regions further out from the core and thus represents an upper limit of any emission model for the total SED.

\subsection{ROTSE-III analysis}

The ROTSE-III (Robotic Optical Transient Search Experiment) array is a world-wide network of four $0.45 \mathrm{~m}$ robotic, automated telescopes built for fast $(\approx 6 \mathrm{~s})$ response to GRB triggers from satellites such as HETE-2 (High Energy Transient Explorer 2) and Swift. The ROTSE-III telescopes have a wide $\left(1.85^{\circ} \times 1.85^{\circ}\right)$ 


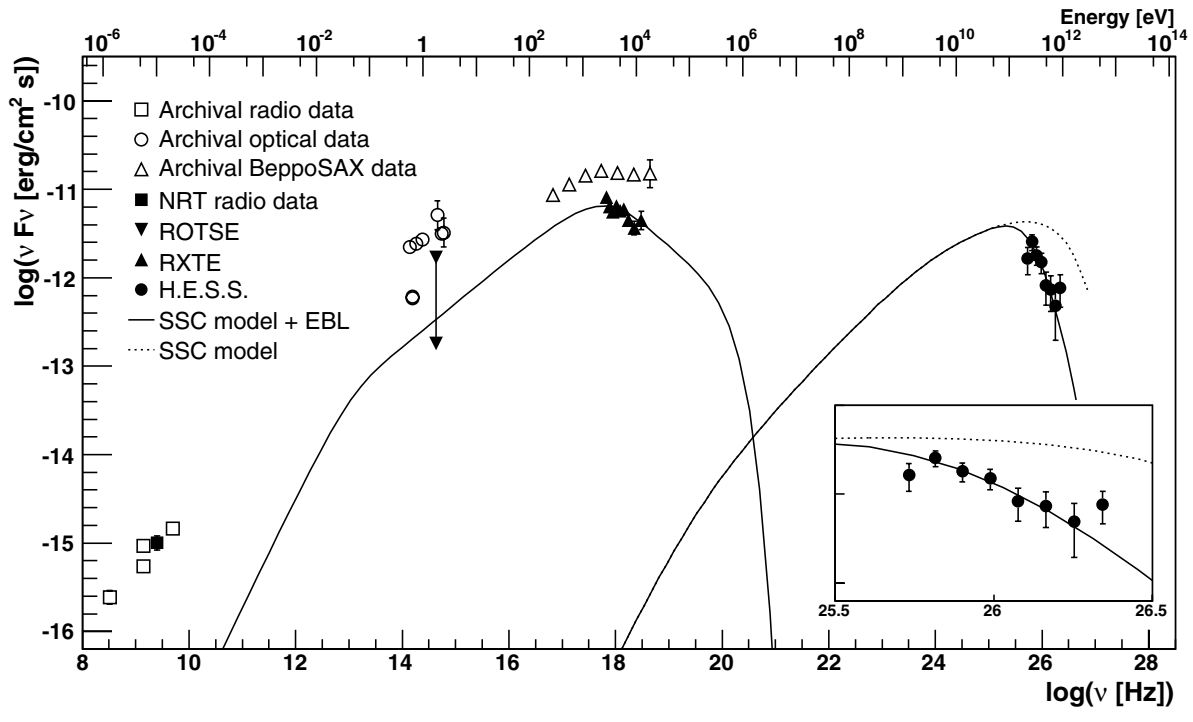

Fig. 4. Spectral energy distribution of H 2356-309. Above $200 \mathrm{GeV}$, the results obtained in this paper are used. Data from NRT, ROTSE-III and RXTE are simultaneous to the HESS observations and shown as filled symbols. All other data are archival and shown as open symbols. The ROTSE-III flux is given after galaxy subtraction. Radio emission arises from regions further out in the jet. A singlezone homogeneous SSC model described in the text is shown as a solid line. The dashed line shows the SSC model without absorption. The inlaid box shows a zoom in the VHE regime.

field of view imaged onto a Marconi $2048 \times 2048$ pixel backilluminated thinned CCD and are operated without filters. The ROTSE-III systems are described in detail in Akerlof et al. (2003). The ROTSE-IIIc telescope, located at the HESS site, has been used to perform an automated monitoring programme of blazars, including H 2356-309. Data is analysed as described in Aharonian et al. (2005c) and references therein. During the observation periods covered by HESS the apparent $R$-band magnitude $m(R)$ from H 2356-309 as measured by ROTSE-III has its maximum at $m(R)=16.1$ and its minimum at $m(R)=16.9$. The host galaxy has been resolved in the optical (Falomo 1991; Scarpa et al. 2000) and near-infrared (Cheung et al. 2003). These observations show that H 2356-309 is a normal elliptical galaxy with an effective radius of about 1.8 arcsec in the $R$ band. The contribution of the galaxy to the observed ROTSE-III flux is estimated to be $m(R)=17$ using a standard de Vaucouleurs radial profile.

\section{Discussion}

In Fig. 4, a broad-band SED obtained from archival data, and simultaneous optical (ROTSE-III) and X-ray (RXTE) data together with the HESS results presented in this paper is shown. Additionally, the result of a simple leptonic model is given as a solid line. The simplest leptonic scenario is a one-zone homogeneous, time independent, synchrotron self-Compton (SSC) model as initially proposed by Jones et al. (1974) for compact non-thermal extragalactic sources. Here, we adopt a description with a spherical emitting region of radius $R$ and homogeneous magnetic field $B$, propagating with Doppler factor $\delta$ with respect to the observer. The high energy electron distribution is described by a broken power law between Lorentz factors $\gamma_{\text {min }}$ and $\gamma_{\max }$, with a break at $\gamma_{\mathrm{b}}$ and a normalisation $K$ (Katarzyński et al. 2001). This SSC scenario is used, taking into account absorption by the EBL, to reproduce the simultaneous data of H 2356-309. The density of the EBL is not well known in the $\mu \mathrm{m}$ wavelength regime. Given the high redshift of H 2356-309 $(z=0.165)$ and a comparatively hard VHE spectrum, important constraints on the EBL density can be derived from the HESS data. This question is addressed in detail in Aharonian et al. (2006a) where constraints on the density of the EBL are derived from HESS observations of 1ES 1101-232 and H 2356-309.
Here, we use the $\mathrm{P} 0.45$ parametrisation from this paper which is very close to the lower limit from galaxy counts.

As shown in Fig. 4, using a model with a reasonable set of parameters provides a satisfactory fit to the simultaneous $\mathrm{X}$-ray and VHE data. The emitting region is characterised by $\delta=18$, $B=0.16 \mathrm{G}$ and $R=3.4 \times 10^{15} \mathrm{~cm}$. The electron power-law distribution is described by $K=1.2 \times 10^{4} \mathrm{~cm}^{-3}, \gamma_{\min }=10^{3}$, $\gamma_{\max }=3 \times 10^{6}$. The Lorentz factor at break energy $\gamma_{\mathrm{b}}$ is located at $\gamma_{\mathrm{b}}=2.5 \times 10^{5}$ to place the peak emission in between optical and X-rays while providing a good fit to the HESS data. We take the canonical index $\alpha_{1}=2$ for the low-energy end and found $\alpha_{2}=4.0$ for the high-energy end so as to fit the observed $\mathrm{X}$-ray power law spectrum. Lowering $\gamma_{\text {min }}$ extends the fit to lower frequencies and enhances IC emission in the $\mathrm{MeV}-\mathrm{GeV}$ domain. Synchrotron self-absorption cuts off emission below IR frequencies when using low values of $\gamma_{\text {min }}$. Radio emission arises from regions further out in the jet. Similar to the case of PKS 2155-304 (Aharonian et al. 2005c) we cannot exclude a possible contribution of such an extended region to the optical flux measured by ROTSE-III. This may soften some of the above-mentioned constraints.

Although our VHE observations provide strong constraints on the physical parameters of single-zone SSC models, there is still some freedom of choice for the parameters that could be constrained further by a better understanding of the origin of the optical emission, a better spectral coverage in the X-ray and sub-TeV region and the observation of possible variability.

\section{Conclusions}

The high frequency peaked BL Lac object H 2356-309, located at a redshift of $z=0.165$, was discovered in the VHE regime by the HESS Cherenkov telescopes. Two different reconstruction and analysis methods were applied to the data both yielding consistent results. No strong evidence for variability in the VHE band is found within the HESS observations. The same holds true in the X-ray band, where the object does not show any strong flux variability, neither in the ASM nor in the pointed observations. Additionally, the RXTE flux, observed simultaneously to the HESS observations, is lower than the previouslymeasured BeppoSAX flux. This might indicate that our observations took place during a relatively low state of emission. 
For the first time, an SED comprising simultaneous radio, optical, X-ray and VHE measurements was made. A simple one-zone SSC model, taking into account absorption by the EBL (Aharonian et al. 2006a), provides a satisfactory description of these data.

Given the high redshift of the object, the observed HESS spectrum provides strong constraints on the density of the EBL (Aharonian et al. 2006a). Future observations of H 2356-309 with HESS will improve the accuracy of the spectral measurement and might also allow an extension of the observed spectrum to higher energies. This will provide further constraints on the absorption of $\gamma$-rays by the EBL.

Acknowledgements. The support of the Namibian authorities and of the University of Namibia in facilitating the construction and operation of HESS is gratefully acknowledged, as is the support by the German Ministry for Education and Research (BMBF), the Max Planck Society, the French Ministry for Research, the CNRS-IN2P3 and the Astroparticle Interdisciplinary Programme of the CNRS, the UK Particle Physics and Astronomy Research Council (PPARC), the IPNP of the Charles University, the South African Department of Science and Technology and National Research Foundation, and by the University of Namibia. We appreciate the excellent work of the technical support staff in Berlin, Durham, Hamburg, Heidelberg, Palaiseau, Paris, Saclay, and in Namibia in the construction and operation of the equipment.

The authors acknowledge the support of the ROTSE-III collaboration. Special thanks also to R. Quimby from the University of Texas for providing tools for data-reduction.

\section{References}

Aharonian, F. A. 2000, New Astron., 5, 377

Aharonian, F. A., et al. (HEGRA Collaboration) 2002, A\&A, 393, 89 Aharonian, F. A., et al. (HEGRA Collaboration) 2003, A\&A, 403, L1 Aharonian, F. A., et al. (HESS Collaboration) 2004, Astropart. Phys., 22, 109 Aharonian, F. A., et al. (HESS Collaboration) 2005a, A\&A, 430, 865 Aharonian, F. A., et al. (HESS Collaboration) 2005b, A\&A, 442, 177 Aharonian, F. A., et al. (HESS Collaboration) 2005c, A\&A, 442, 895 Aharonian, F. A., et al. (HESS Collaboration) 2005d, in preparation Aharonian, F. A., et al. (HESS Collaboration) 2006a, Nature, 440, 1018 Aharonian, F. A., et al. (HESS Collaboration) 2006b, A\&A, 449, 223 Akerlof, C. W., Kehoe, R. L., McKay, T. A., et al. 2003, PASP, 115, 132 Cheung, C. C., Urry, C. M., Scarpa, R., \& Giavalisco, M. 2003, ApJ, 599, 155 Costamante, L., \& Ghisellini, G. 2002, A\&A, 384, 56

Costamante, L., Ghisellini, G., Giommi, P., et al. 2001, A\&A, 371, 512

Falomo, R. 1991, AJ, 101, 821

Forman, W., Jones, C., Cominsky, L., et al. 1978, ApJS, 38, 357

Fossati, G., Maraschi, L., Celotti, A., et al. 1998, MNRAS, 299, 433

Ghisellini, G., Celotti, A., \& Costamante, L. 2002, A\&A, 386, 833

Hinton, J. A. 2004, New Astron. Rev., 48, 331

Jahoda, K., \& PCA Team 1996, BAAS, 28, 1285

Jones, T. W., O’dell, S. L., \& Stein, W. A. 1974, ApJ, 188, 353

Katarzyński, K., Sol, H., \& Kus, A. 2001, A\&A, 367, 809

Krawczynski, H., Sambruna, R., Kohnle, A., et al. 2001, ApJ, 559, 187

Lemoine-Goumard, M., \& Degrange, B. 2004, AIP Conf. Proc., 745, 697

Lemoine-Goumard, M., Degrange, B., \& Tluczykont, M. 2006, Astropart. Phys., 25,195

Li, T. P., \& Ma, Y. Q. 1983, ApJ, 272, 317
Mannheim, K. 1993, A\&A, 269, 67

Mücke, A., \& Protheroe, R. J. 2001, Astroparticle Physics, 15, 121

Piron, F. 2000, Ph.D. Thesis, Université de Paris XI

Pohl, M., \& Schlickeiser, R. 2000, A\&A, 354, 395

Primack, J. R, Bullock, J. S, Summerville, R. S, \& MacMinn, D. 1999, Astroparticle Physics, 11, 93

Punch, M., Akerlof, C. W., Cawley, M. F., et al. 1992, Nature, 358, 477

Rees, M. J. 1984, ARA\&A, 22, 471

Scarpa, R., Urry, C. M., Padovani, P., Calzetti, D., \& O’Dowd, M. 2000, ApJ, 544,258

Schwartz, D., Brissenden, R. J. V., Thuoy, I. R., et al. 1989, in Lecture Notes in Physics, ed. L. Maraschi, T. Maccacaro, \& M.-H. Ulrich (Berlin: Springer), 334,211

Sikora, M., \& Madejski, G. M. 2001, in American Institute of Physics Conference Series, 558, 275

Stecker, F. W., de Jager, O. C., \& Salamon, M. H. 1992, ApJ, 390, L49

Urry, C. M., \& Padovani, P. 1995, PASP, 107, 803

van Driel, W., Pezzani, J., \& Gerard, E. 1996, in High-Sensitivity Radio Astronomy, 229

Wood, K. S., Meekins, J. F., Yentis, D. J., et al. 1984, ApJS, 56, 507

1 Max-Planck-Institut für Kernphysik, Heidelberg, Germany

2 Yerevan Physics Institute, Yerevan, Armenia

3 Centre d'Étude Spatiale des Rayonnements, CNRS/UPS, Toulouse, France

4 Universität Hamburg, Institut für Experimentalphysik, Hamburg, Germany

5 Institut für Physik, Humboldt-Universität zu Berlin, Germany

6 LUTH, UMR 8102 du CNRS, Observatoire de Paris, Section de Meudon, France

7 University of Durham, Department of Physics, Durham, UK

8 Unit for Space Physics, North-West University, Potchefstroom, South Africa

9 Laboratoire Leprince-Ringuet, IN2P3/CNRS, École Polytechnique, Palaiseau, France e-mail: martin.tluczykont@poly.in2p3.fr

10 APC, Paris, France (UMR 7164, CNRS, Université Paris VII, CEA, Observatoire de Paris)

11 Dublin Institute for Advanced Studies, Ireland

12 Landessternwarte, Universität Heidelberg, Germany

13 Laboratoire de Physique Théorique et Astroparticules, IN2P3/CNRS, Université Montpellier II, CC 70, France

14 DAPNIA/DSM/CEA, CE Saclay, France

15 Laboratoire d'Astrophysique de Grenoble, INSU/CNRS, Université Joseph Fourier, France

16 Institut für Astronomie und Astrophysik, Universität Tübingen, Germany

17 Laboratoire de Physique Nucléaire et de Hautes Énergies, IN2P3/CNRS, Universités Paris VI \& VII, France

18 Institute of Particle and Nuclear Physics, Charles University, Prague, Czech Republic

19 Institut für Theoretische Physik, Lehrstuhl IV: Weltraum und Astrophysik, Ruhr-Universität Bochum, Germany

20 University of Namibia, Windhoek, Namibia

21 European Associated Laboratory for Gamma-Ray Astronomy, jointly supported by CNRS and MPG

22 Now at DESY, Platanenallee 6, 15738 Zeuthen, Germany 